\title{
Analysis of the Load-Stress Response Characteristics of the Bogie Frame in Intercity Electric Multiple Unit
}

\author{
Hua Zou*, Shou-Guang Sun, Qiang Li and Zun-Song Ren
}

\begin{abstract}
Load spectra research for bogie frame requires establishing the load-stress relationship on working condition, which has been omitted by the researchers. With the load-stress of the bogie frame of an intercity Electric Multiple Unit (Hereinafter referred to as EMU) as the research object, an optimization model of the load-stress transfer relationship is established. The load-stress coefficient for EMU bogie frame was calibrated in the laboratory bench and online test was arranged on Dazhou-Chengdu line. Comparison of nonlinear and linear neural networks proves that the linear transitive relation between the load and stress of the bogie frame in the operating process is highly suitable. An optimization model of the load-stress transfer coefficient is obtained. The data calculated with the modified coefficient are closer to the dynamic stress results in the actual operating process than the data calculated with the calibration coefficient. The coefficient of the modified transitive relation is unaffected by operating area, empty load, heavy load, or other conditions in the operating process of the intercity EMU. The real loads in actual situations are obtained. The model of online load-stress relationship that is highly suitable for line stress calculation is finally established. The research is helpful for further damage calculation and inferring the time history signal of the load in load spectra research.
\end{abstract}

Keywords: Bogie frame, Load, Stress, Transitive relation, Neural network

\section{Introduction}

The study of load spectrum is the hotspot of the current bogie frame research [1-3]. How to obtain the load spectrum of the bogie frame, there are many researchers have done a lot of work. How to describe the relationship between load and stress under on-line conditions to calculate the damage consistency is lack of attention from the researchers at present. The load spectrum of a bogie is usually obtained with the method of measured load spectrum. It can also be obtained with the multi-body system dynamics simulation method. Another method to obtain the load spectrum is to use the correlation among the power spectral densities (PSDs) of time domain signals.

\footnotetext{
*Correspondence: hzou@bjtu.edu.cn

Engineering Research Center of Structure Reliability and Operation

Measurement Technology of Rail Guided Vehicles of Ministry

of Education, Beijing Jiaotong University, Beijing 100044, China
}

Wang et al. [4] studied the load spectra of high-speed train bogies by conducting a long-term on-track test on the Wuhan-Guangzhou passenger line.The research showed that wheel profiling improves the loading condition of the bogie frame. However, the transfer relation between load and stress was not discussed in the study because of the different working conditions. Zhu et al. [5] conducted a theoretical study on and an experimental validation of elastic dynamic load spectra on the bogie frame of a high-speed train. The computed result proved that the simplified load series is reasonable. The contribution of modal stress to the overall damage is small. However, the overall damage in the line test was calculated with a laboratory bench calibration coefficient. Ren et al. [6] presented methods to measure the axle spring load, trailing arm seat lateral force and dynamic stresses of powered and non-powered bogies of a $350 \mathrm{~km} / \mathrm{h}$ EMU. The characteristics of the forces and the stresses for the EMU were investigated by Hilbert transform of the
Springer Open

(c) The Author(s) 2018. This article is distributed under the terms of the Creative Commons Attribution 4.0 International License (http://creativecommons.org/licenses/by/4.0/), which permits unrestricted use, distribution, and reproduction in any medium, provided you give appropriate credit to the original author(s) and the source, provide a link to the Creative Commons license, and indicate if changes were made. 
sample data. The characteristics of the measured loads are useful to establish load conditions for laboratory tests of bogie's fatigue assessment. But the relationship of loan-stress has not been given out in the research.

Mi et al. [7] used load-stress linear transfer relations to calculate stress. A line test dynamic load spectrum was obtained as an input by using the finite element method to calculate line stress. The maximum calculated equivalent stress was similar to the measured equivalent stress. However, the difference between time domain stress calculated by the load and the measured stress was not discussed. Ma et al. [8] combined simulations with multibody dynamics and partial experimental data to obtain axle box dynamic loads. Given the differences and limitations in line conditions, operating conditions, and vehicle model types, the method cannot fully and accurately reflect structural load characteristics under practical conditions.

Wolfsteiner et al. [9] presented an alternative method that estimates load spectra directly from the PSDs of the corresponding time signals. However, the load-strain relationship was not presented.

Research on the load spectrum of the bogie frame can adopt multi-body system dynamics simulation to obtain the main load of the bogie. Rigid and elastic bogie frames are used to establish a model for multi-body system dynamics. Simulation calculation indicates that the loads of rigid and elastic bogie frames possess similar maximum dynamic amplitude; however, the function frequency of a bogie with an elastic frame is significantly higher than that of a bogie with a rigid frame within a different dynamic amplitude range of the main load $[10,11]$. If a bogie frame is regarded as a rigid body, its load-stress transitive relation is linear, and the load-stress coefficient obtained with a quasi-static method can be employed as a reference for research on the load spectrum [12]. If a bogie frame is regarded as an elastic body [13-15], a transient analysis can be conducted, and the input could include modal information coupled with nonlinear processing of the wheel-rail relationship [16-19]. As a result, the transitive relation between the measured load and stress is nonlinear and thus requires the adoption of a nonlinear fitting method for optimized fitting of the multi-input model.

Although the method of measured load spectrum is costly, it can effectively reflect the actual load of the line and the relationship between load and stress. In this study, the bogie frame of an intercity EMU was adopted as the research object to obtain online load and stress data. Two different transitive relations were compared. An optimized, simplified model was established, and the modified linear transitive relation coefficient of load and stress under the condition of one running line was obtained. The dynamic stress data obtained with the modified transitive relation were significantly similar to the dynamic stress results in the actual operation. The coefficient of the modified transitive relation was unaffected by the operating conditions of the intercity EMU, such as speed, operating area, and empty or loaded condition.

The intercity EMU under study is a new motor train unit independently researched and designed by China for smooth microcirculation between cities. The unit operates in modified lines and on a non-ballasted track. Its maximum operating speed is $200 \mathrm{~km} / \mathrm{h}$.

\section{Quasi-Static Calibration Coefficient}

Quasi-static load-stress calibration is performed in a laboratory to study the load-stress transitive relation of the intercity EMU. The calibration site is shown in Figure 1. The assembly of the bogie frame follows the technical requirements of its actual operating condition, and the calibration load system is shown in Figure 2.

The bogie frame has many load systems, such as sinkfloat, side roll, torsion, lateral, motor bracket, gearbox bracket, anti-yaw, wheel brake, and traction loads. A global load and key stress points are adopted to analyze the transitive relation and completely verify the idea proposed in this paper. The measuring points utilized include D13, D15, D26, D34, D46, D48, D55, and D57, which represent key response points in various regions. The specific locations of these points are shown in Figure 3.

The calibration data of the laboratory bench are shown in Table 1.

As shown in Table 1, all eight selected measuring points have different response factors for all loads. Therefore, a closed computational model composed of eight stress points and nine loads is formed. The calculation methods for sink-float, side roll, and torsion loads are presented in a previous study [1]; the other loads are obtained with component sensors, which are calibrated by grouping the bridge circuits consisting of various sensitive points in

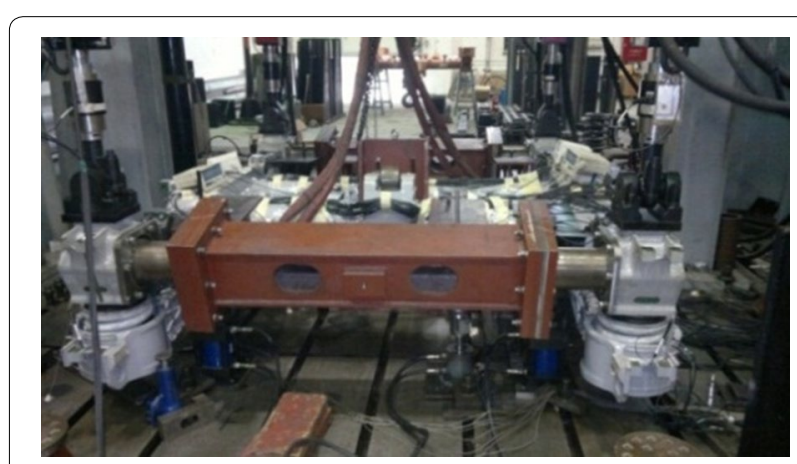

Figure 1 Bogie frame calibration site 


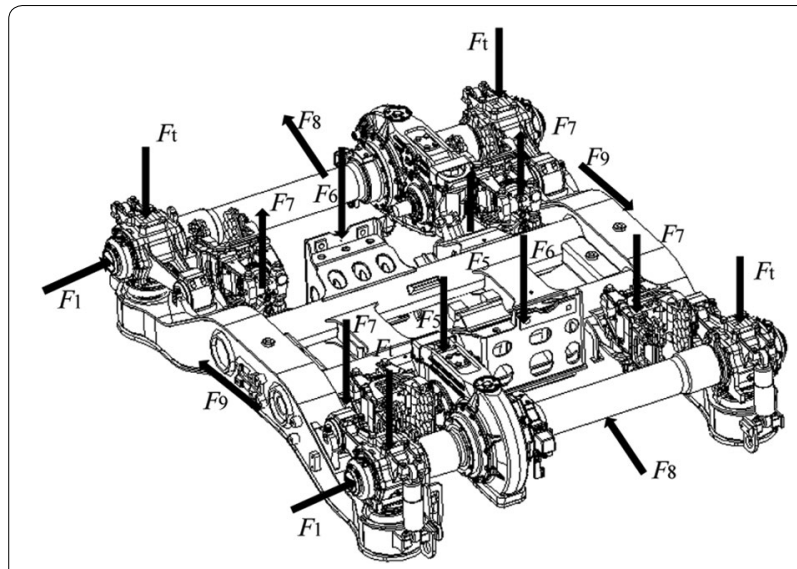

Figure 2 Bogie frame load distribution each component part [20]. Repetitious data are not presented in the current paper.

\section{Online Test}

The calibrated and well-assembled bogie frame is mounted on the intercity EMU; the operating area is Dazhou-Chengdu. Given that the online test involves other test projects, the braking system signals are removed, and the wheel braking coefficient of the transitive relation matrix is ignored.

The test bench indicates that the transitive relation load-stress response is

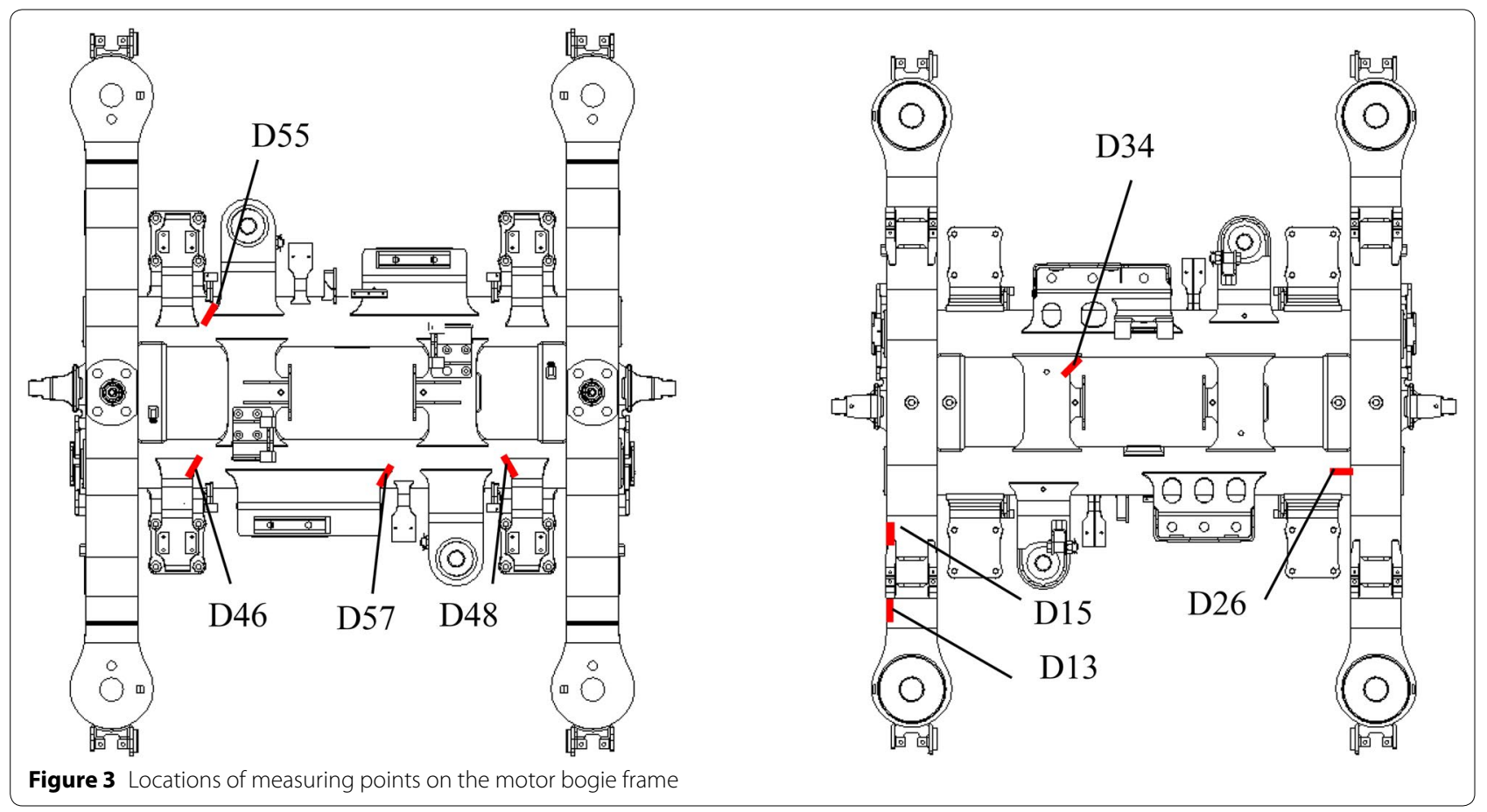

Table 1 Calibrated load-stress data on laboratory bench $\mu \varepsilon$

\begin{tabular}{lrrrrrrrrr}
\hline Measuring point & \multicolumn{1}{c}{$\boldsymbol{F}_{\mathbf{1}}$} & \multicolumn{1}{c}{$\boldsymbol{F}_{\mathbf{2}}$} & \multicolumn{1}{c}{$\boldsymbol{F}_{\mathbf{3}}$} & \multicolumn{1}{c}{$\boldsymbol{F}_{\mathbf{4}}$} & $\boldsymbol{F}_{\mathbf{5}}$ & $\boldsymbol{F}_{\mathbf{6}}$ & $\boldsymbol{F}_{\mathbf{7}}$ & $\boldsymbol{F}_{\mathbf{8}}$ & $\boldsymbol{F}_{\mathbf{9}}$ \\
\hline D13 & 11 & 43 & 49 & -52 & 19 & 0 & 26 & -3 & -2 \\
D15 & 25 & 71 & 76 & -70 & 26 & 7 & 36 & 15 \\
D26 & -28 & -8 & 2 & 116 & -60 & 30 & -78 & -10 & -18 \\
D34 & 2 & 7 & 2 & -73 & 7 & -15 & 3 & 34 & 26 \\
D46 & 1 & -2 & -9 & -63 & 13 & 128 & -114 & 7 & 7 \\
D48 & -1 & -1 & 5 & 58 & 61 & 89 & -104 & 6 \\
D55 & 14 & 13 & 14 & -98 & 71 & -14 & -52 & -20 & -14 \\
D57 & -20 & 4 & 3 & 62 & -28 & 72 & 35 & -1 & 4 \\
\hline
\end{tabular}

$F_{1}$ lateral load is $30 \mathrm{kN}, F_{2}$ sink-float load is $12 \mathrm{kN}, F_{3}$ side roll load is $12 \mathrm{kN}, F_{4}$ torsion load is $12 \mathrm{kN}, F_{5}$ vertical gearbox bracket load is $30 \mathrm{kN}, F_{6}$ vertical motor bracket load is $40 \mathrm{kN}, F_{7}$ wheel brake load is $15 \mathrm{kN}, F_{8}$ longitudinal load is $30 \mathrm{kN}$, and $F_{9}$ anti-yaw load is $20 \mathrm{kN}$ 


$$
\left(\begin{array}{l}
\text { D13 } \\
\text { D15 } \\
\text { D26 } \\
\text { D34 } \\
\text { D46 } \\
\text { D48 } \\
\text { D55 } \\
\text { D57 }
\end{array}\right)=\left(\begin{array}{cccccccc}
0.18 & 3.58 & 4.08 & -4.33 & 0.63 & 0 & -0.10 & -0.10 \\
0.42 & 5.92 & 6.33 & -5.83 & 0.87 & 0.18 & 0.50 & -0.05 \\
-0.47 & -0.67 & 0.17 & 9.67 & -2.00 & 0.75 & -0.33 & -0.90 \\
0.03 & 0.58 & 0.17 & -6.08 & 0.23 & -0.38 & 1.13 & 1.30 \\
0.02 & -0.17 & -0.75 & -5.25 & 0.43 & 3.20 & 0.23 & 0.35 \\
-0.02 & -0.08 & 0.42 & 4.83 & 2.03 & 2.23 & 0.20 & 0.25 \\
0.23 & 1.08 & 1.17 & -8.17 & 2.37 & -0.35 & -0.67 & -0.70 \\
-0.33 & 0.33 & 0.25 & 5.17 & -0.93 & 1.80 & -0.03 & 0.20
\end{array}\right) \times\left(\begin{array}{c}
F_{1} \\
F_{2} \\
F_{3} \\
F_{4} \\
F_{5} \\
F_{6} \\
F_{8} \\
F_{9}
\end{array}\right)
$$

A group of test signals of the test run with a speed of $160 \mathrm{~km} / \mathrm{h}$ are selected. The frequency spectral maps of some of the original signals are shown in Figures 4, 5, 6, 7 .

As shown in the frequency spectral maps, the frequency spectra are mainly concentrated in the low- and mediumfrequency responses below $100 \mathrm{~Hz}$. This result is consistent with that in other studies [21-23]. The frequency spectrum of the motor bracket load is concentrated at 20 and $60 \mathrm{HZ}$. The frequency spectrum of the gearbox bracket load is concentrated below $10 \mathrm{~Hz}$. The torsion load spectrum is concentrated below $10 \mathrm{~Hz}$. The frequency spectrum of the stress response is concentrated at 20 and $60 \mathrm{~Hz}$. Accordingly, a $100 \mathrm{~Hz}$ low-pass filter and a $50 \mathrm{~Hz}$ band-pass filter are adopted to remove some random disturbances and power-frequency interferences.

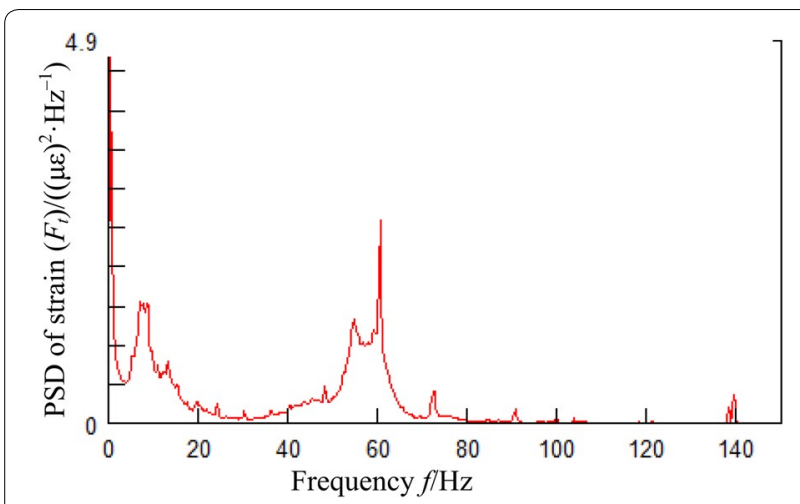

Figure 4 Motor bracket load frequency spectrum

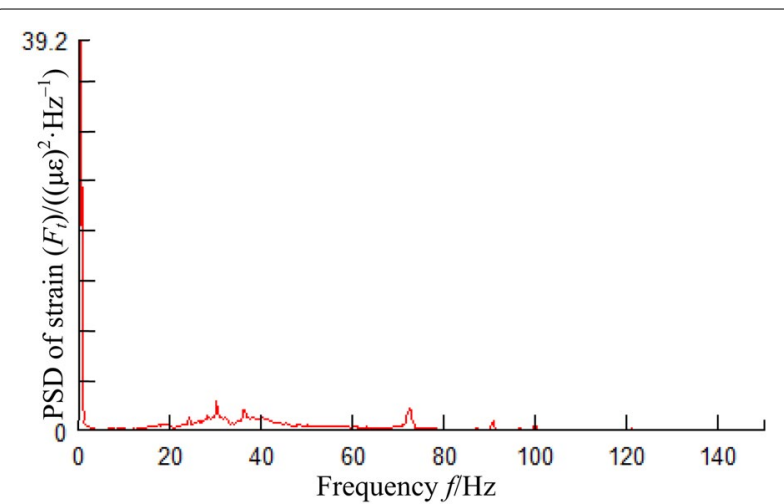

Figure 5 Gearbox bracket load frequency spectrum
If the load-stress response characteristics satisfy a linear relationship, then the stress value can be calculated using the load value, that is, the stress value is the product of the filtered load signal and the corresponding transitive relation coefficient. Subsequently, the calculated stress value and the actual measured stress value can be compared. In Figure 8, the calculated value of the assumed linear response at measuring point D46 is smaller than the measured value (blue line covers the red line; difference will be shown in Table 2). In Figure 9, the calculated value of the assumed linear response at measuring point $\mathrm{D} 48$ is greater than the measured value (red line covers the blue line; difference will be shown in Table 2).

\section{Fitting Evaluation Method}

Given that the adopted frequency is $1000 \mathrm{~Hz}$ and the maximum speed is maintained within $200 \mathrm{~km} / \mathrm{h}$, a $400 \mathrm{~km}$ run takes approximately $3 \mathrm{~h}$, and the amount of sample data is approximately $10^{7}$. For simplification, the stress distribution evaluation method can be adopted for the fitting evaluation of such a large amount of data. However, the stress distribution function has no chronological order; consequently, it has poor relativity with the time-domain signal. Thus, this paper adopts percentage mean square error (PMSE) for fitting evaluation.

$$
e(n)=(d(n)-x(n)) / x(n),
$$

where $d(n)$ is the calculated stress and $x(n)$ is the actual measured signal. Values that are less than 2 are disregarded to eliminate the interference error of the 0 value signal.

PMSE, expressed as follows, is adopted as an evaluation index:

$$
\operatorname{PMSE}=\frac{1}{N} \sum_{k=1}^{N} e^{2}(k) \pi,
$$

where $N$ is the fitting sample size.

The fitting evaluation method can be utilized to identify the difference between the stress value calculated using the calibration coefficient and the actual stress value in the operation process. 


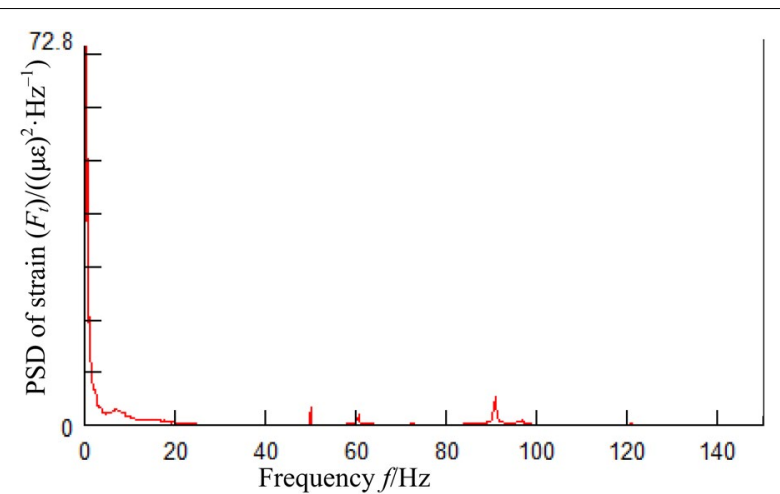

Figure 6 Torsion load frequency spectrum

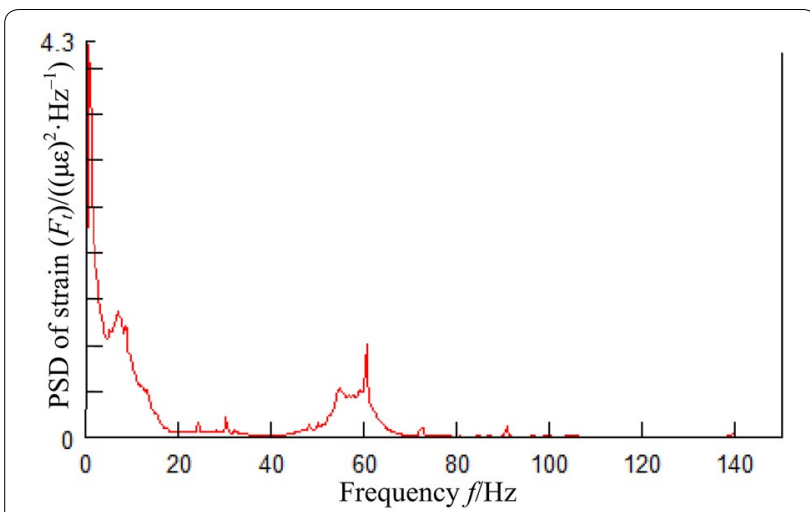

Figure 7 Stress frequency spectrum of measuring point D46

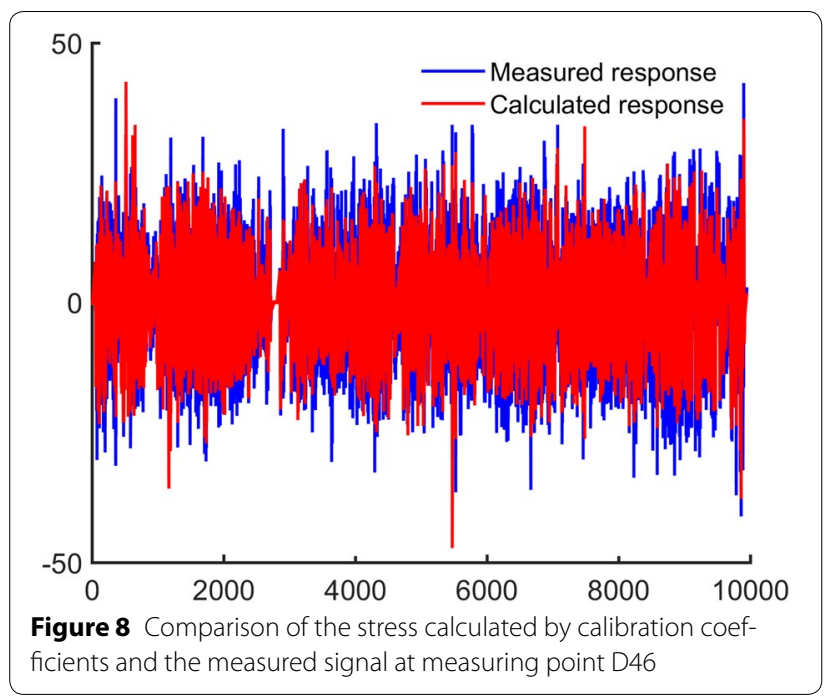

The fitting error of each stress calculated according to the load time history signal and the load-stress calibration coefficient is shown in Table 2.

\section{Neural Network Fitting}

According to different transfer functions, the neural network can be divided into single-layer perceptron neural network, linear neural network, multi-node BP neural network, RBF/GRNN network, Hopfield neural network, random neural network, and so on. Single-layer perceptron neural networks are mainly utilized to solve binary pattern recognition problems. Linear neural networks can be used for simple linear separable stress values and linear fitting. BP networks can be employed for linear non-separable pattern recognition, function fitting, and optimization. RBF/GRNN networks can be used for the function approximation of small samples. Hopfield networks can be employed to solve complex pattern recognition problems and achieve prediction and optimization in time domain [22].

The RBF/GRNN network trains a number of network nodes according to sample size [24, 25]. If the sample size is larger than 105, the network will suffer from heavy operational burden, and a space overflow error will occur. Given that the amount of sample data obtained through the test is 107, an optimized BP neural network is adopted in this study for calculation.

The BP network algorithm is the most widely used algorithm for perceptron networks [26]; approximately $80 \%$ of neural network models adopt a BP network or a modification of a BP network. A multilayer perceptron network usually consists of three parts: input, hidden, and output layers. The input and output layers have one layer, whereas the hidden layer may have multiple layers. Currently, the two-layer hidden network is the most widely used network [26]; its structure is shown in Figure 10. For a multi-layer feedforward network, the number of nodes in a hidden layer is the key to success. If the number of nodes is too small, the network cannot obtain sufficient information to solve the problem; if the number of nodes is too large, the training time will be increased. More importantly, too many nodes in a hidden layer may lead to the so-called "overfitting" problem, that is, the test error will increase and lead to reduced fitting capability. Therefore, the number of nodes in a hidden layer should be rationally selected. Deciding on the number of hidden layers and nodes in a hidden layer is a complex task, but the general principle is to select a few nodes in a hidden layer to correctly reflect the input-output relationship and simplify the network structure. In this study, a network structure incremental pattern is adopted. That is, a few nodes are set to train the network and test the learning error. The number of nodes is then gradually increased until the learning error has no significant reduction.

The transfer function and number of network nodes are adopted as parameters to compare nonlinear and linear optimization. 
Table 2 Calculated stress values and the measured signal fitting error

\begin{tabular}{lllllllll}
\hline Error & \multicolumn{9}{l}{ Measuring points } \\
\cline { 2 - 9 } & D13 & D15 & D26 & D34 & D46 & D48 & D55 & D57 \\
\hline Mse1 & 0.36 & 2.26 & 1.88 & 1.97 & 2.54 & 1.63 & 0.84 & 1.74 \\
\hline
\end{tabular}

Mse1 represents the percentage mean square error between the stress value calculated with Eq. (1) and the measured stress value

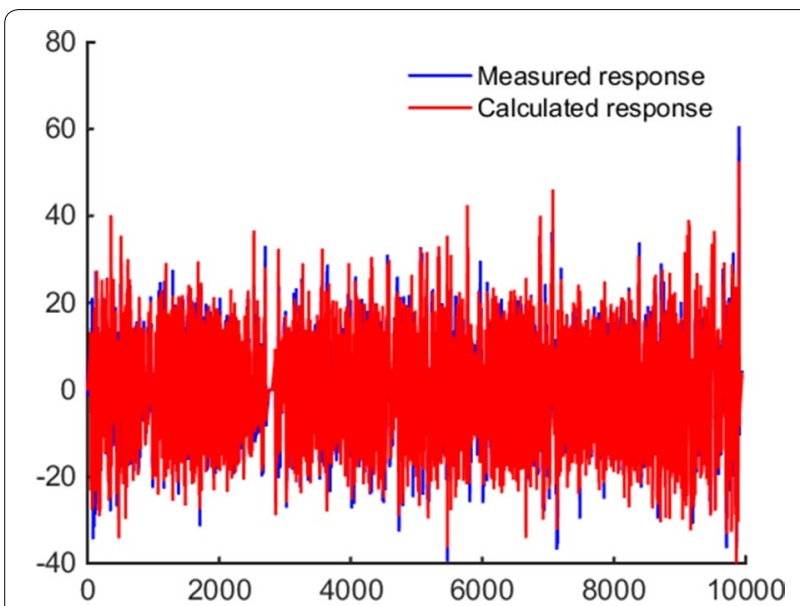

Figure 9 Comparison of the stress calculated by calibration coefficients and the measured signal at measuring point D48

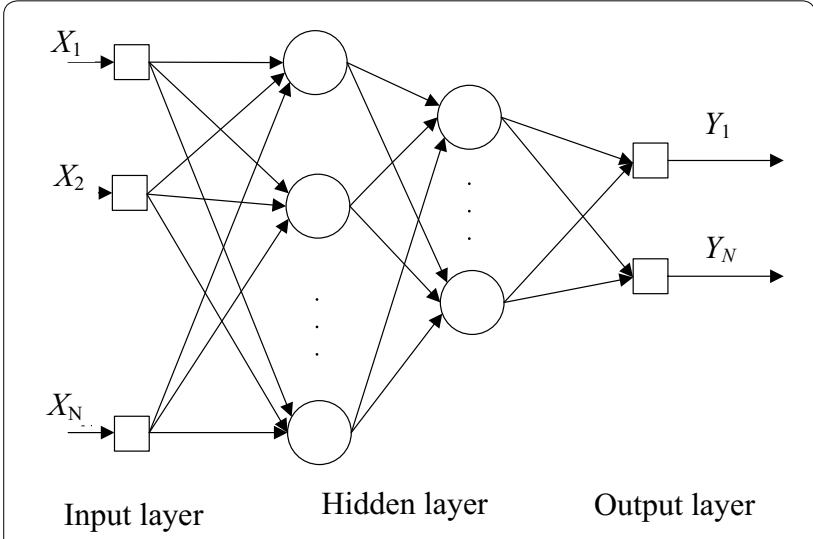

Figure 10 Typical topology of a neural network

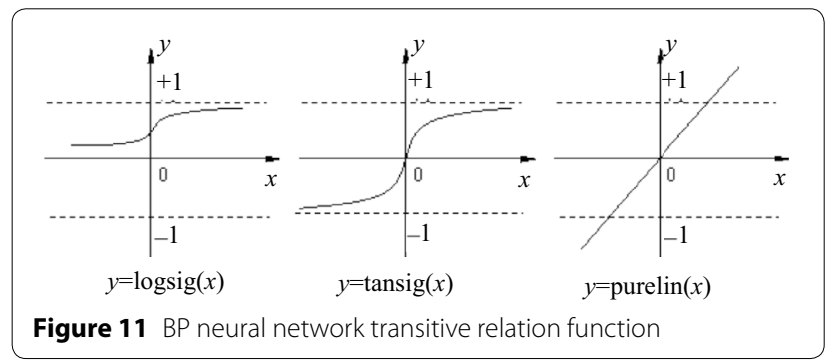

The transfer function can be divided into three categories, as shown in Figure 11. Nonlinear logsig function is expressed as Eq. (4), and nonlinear tansig is expressed as Eq. (5). The linear transfer function is purelin, i.e., $y=x$, whose linear coefficient is the coefficient of the network node.

$$
\begin{aligned}
& \log \operatorname{sig}(n)=\frac{1}{1+\exp (-n)}, \\
& \operatorname{tansig}(n)=\frac{2}{1+\exp (-2 n)}-1 .
\end{aligned}
$$

The calculation method is shown in the flowchart in Figure 12.

With D46 as the calculation object, the nonlinear network has eight inputs (lateral, sink-float, side roll, torsion, gearbox bracket, motor bracket, longitudinal, and anti-yaw loads) and one stress point as the output. Part of the data calculated in the optimization process is listed in Table 3. As shown in Table 3, the optimal result is that all hidden layers form a linear transitive relation network.

The optimal solution is the network structure with a linear transitive relation. The coefficient of the linear input layer with one node and the first layer of the hidden layer is

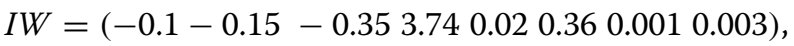

The coefficient of the first linear hidden layer and the second linear hidden layer is

$$
L W=-1.23 \text {. }
$$

Therefore, the transitive relation between the input layer, which includes eight input loads, and the output layer at the $\mathrm{D} 46$ measuring point is

$$
\mathrm{D} 46=I W \times L W \times\left(\begin{array}{c}
F_{1} \\
F_{2} \\
F_{3} \\
F_{4} \\
F_{5} \\
F_{6} \\
F_{8} \\
F_{9}
\end{array}\right),
$$

$$
\mathrm{D} 46=\left(\begin{array}{r}
0.12 \\
0.19 \\
0.43 \\
-4.62 \\
-0.03 \\
-0.44 \\
-0.001 \\
-0.004
\end{array}\right)^{\mathrm{T}} \times\left(\begin{array}{l}
F_{1} \\
F_{2} \\
F_{3} \\
F_{4} \\
F_{5} \\
F_{6} \\
F_{8} \\
F_{9}
\end{array}\right) .
$$




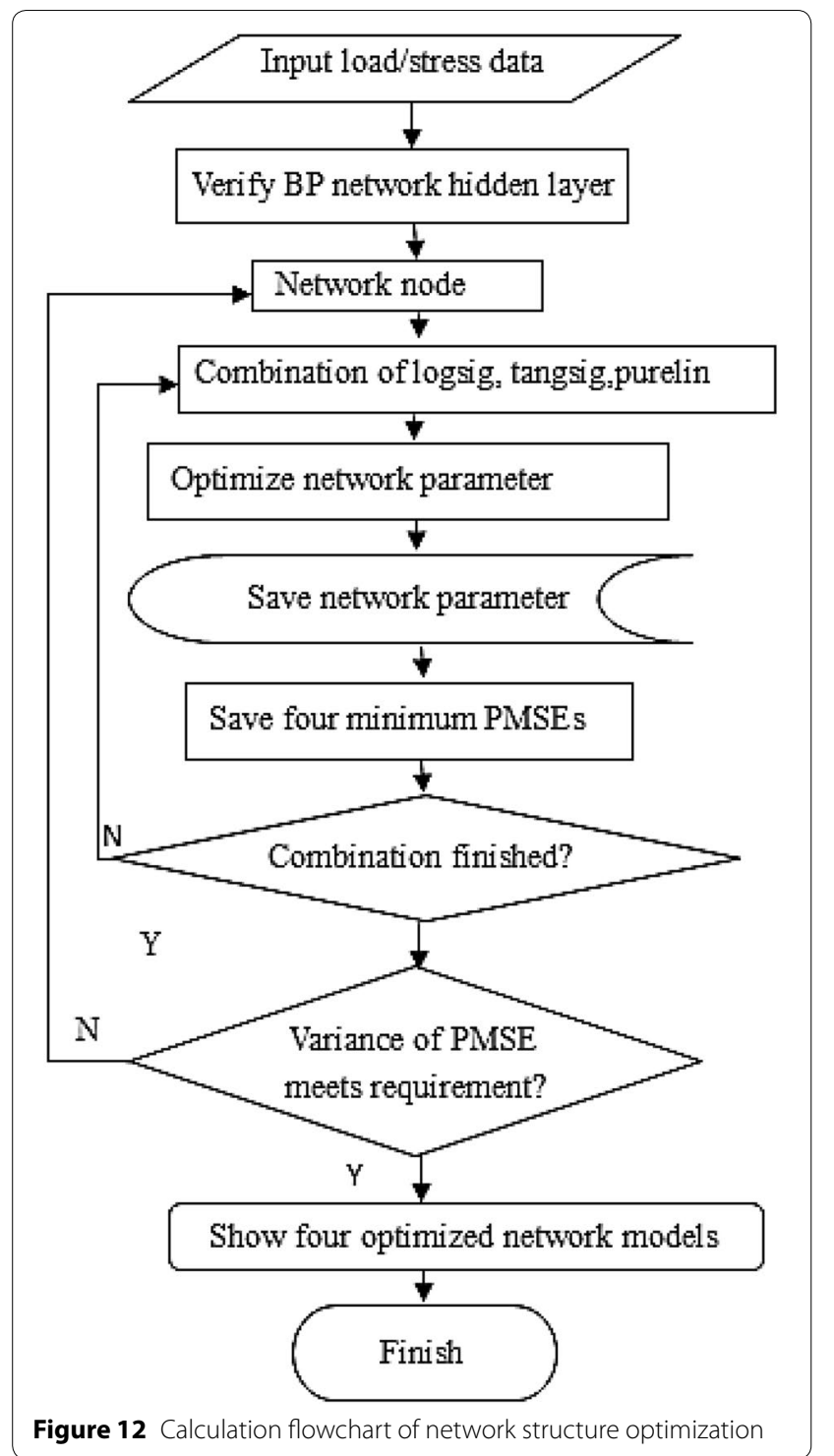

Table 3 Network parameters and corresponding calculation error and calculation time

\begin{tabular}{|c|c|c|c|c|}
\hline \multirow{2}{*}{$\begin{array}{l}\text { Two hidden layer } \\
\text { transitive relation }\end{array}$} & \multicolumn{2}{|c|}{ One node } & \multicolumn{2}{|c|}{ Two nodes } \\
\hline & Error & $\begin{array}{l}\text { Calculation } \\
\text { time } t(\mathrm{~s})\end{array}$ & Error & $\begin{array}{l}\text { Calculation } \\
\text { time } t(\mathrm{~s})\end{array}$ \\
\hline 'tansig' $\times$ 'tansig' & 0.20 & 5010 & 0.20 & 4148 \\
\hline 'logsig' × 'tansig' & 0.20 & 4163 & 0.20 & 4991 \\
\hline 'purelin' $\times$ 'tansig' & 0.20 & 4977 & 0.20 & 4150 \\
\hline 'tansig' $\times$ 'logsig' & 0.20 & 4143 & 0.20 & 4959 \\
\hline 'logsig'× 'logsig' & 0.22 & 4985 & 0.20 & 4823 \\
\hline 'purelin'× 'logsig' & 0.20 & 4671 & 0.20 & 4983 \\
\hline 'tansig' × 'purelin' & 0.08 & 1184 & 0.08 & 1686 \\
\hline 'logsig' × 'purelin' & 0.07 & 1034 & 0.07 & 1685 \\
\hline 'purelin'× 'purelin' & 0.06 & 103 & 0.06 & 767 \\
\hline
\end{tabular}

The operating environment is MATLAB R2014 run on an Intel (R) Core (TM) 17-4790 CPU with $3.6 \mathrm{GHz}$ processor and $16 \mathrm{~GB}$ RAM

As shown in the process in Figure 13, the transitive relation coefficient between the stress at D46 and the eight loads is expressed as Eq. (9), which is optimized by the linear neural network. Similarly, a single-layer network computational method can be utilized to obtain the transitive relation coefficients between D13, D15, D26, D34, D48, D55, and D57 and the eight input loads.

A set of calculated stress values can be obtained by using the modified transitive relation in Eq. (10). The optimized stress errors are shown in Table 4. The local area model is zoomed in in Figure 14. The differences among the actual measured, unmodified calculated, and optimized calculated signals are clear. Compared with the unmodified calculated signal, the optimized calculated signal fits the actual measured signal more.

$$
\left(\begin{array}{l}
\text { D13 } \\
\text { D15 } \\
\text { D26 } \\
\text { D34 } \\
\text { D46 } \\
\text { D48 } \\
\text { D55 } \\
\text { D57 }
\end{array}\right)=\left(\begin{array}{cccccccc}
0.04 & 3.83 & 4.20 & -4.05 & -0.12 & -0.21 & -0.01 & 0.002 \\
-0.19 & 4.98 & 5.64 & -4.70 & -0.19 & -0.26 & -0.005 & 0.03 \\
-0.37 & -2.23 & -3.33 & 9.54 & 0.14 & 0.53 & -0.0001 & 0.003 \\
-1.18 & 0.42 & 0.21 & -5.58 & -0.11 & 0.06 & -0.08 & 0.01 \\
0.12 & 0.19 & 0.43 & -4.62 & -0.03 & -0.44 & -0.001 & -0.004 \\
-0.37 & -1.34 & -0.61 & 5.79 & -0.21 & -0.12 & -0.02 & 0.01 \\
-0.07 & 1.71 & 2.56 & -8.61 & -0.18 & -0.36 & 0.06 & -0.0002 \\
1.01 & -1.10 & -0.05 & 4.45 & 0.10 & -0.04 & 0.01 & -0.02
\end{array}\right) \times\left(\begin{array}{l}
F_{1} \\
F_{2} \\
F_{3} \\
F_{4} \\
F_{5} \\
F_{6} \\
F_{8} \\
F_{9}
\end{array}\right),
$$

Therefore, the neural network that contains two hidden layers can be converted into a single-layer linear network, the topology structure of which is shown in Figure 13.

The transitive relation of the two-node linear network structure can be simplified into a one-node single-layer linear network through the same method.

\section{Linear Transitive Relation Significance Test}

Mathematical Model Description [27]: If $k$ factors, $X_{1}, X_{2}$, ..., $X_{k}$, exist, and dependent variable $y$ has the following linear relationship, then 


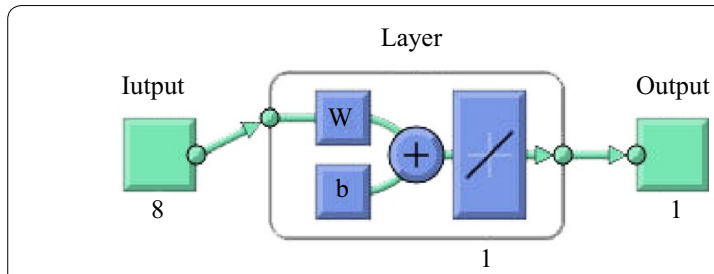

Figure 13 Single-layer linear network

$$
\begin{aligned}
& f\left(x_{1}, x_{2}, \ldots, x_{k}\right)=y \\
& \quad=\beta_{1} x_{1}+\ldots+\beta_{k} x_{k}+\varepsilon \quad \varepsilon \sim N\left(0, \sigma^{2}\right) .
\end{aligned}
$$

Equation (11) is a multiple linear regression function, where $\beta_{i}(i=1,2, \ldots, k)$ is called the regression coefficient. If all $X_{1}, X_{2}, \ldots, X_{k}$ have no significant influence on $y$, the coefficient in the model (Eq. (11)) is $\beta_{i}=0(i=1,2, \ldots, k)$. The significance test aims to assess whether

$$
\mathrm{H}_{0}: \beta_{1}=\beta_{2}=\ldots=\beta_{k}=0 .
$$

The three error statistics are total deviation square sum $S_{T}^{2}=\sum_{i=1}^{n}\left(y_{i}-\bar{y}\right)^{2}$, residual sum of squares $S_{E}^{2}=\sum_{i=1}^{n}\left(y_{i}-\hat{y}_{i}\right)^{2}$, and the regression sum of squares $S_{R}^{2}=\sum_{i=1}^{i=1}\left(\hat{y}_{i}-\bar{y}\right)^{2} . \quad$ If $\quad \beta_{1}=\beta_{2}=\ldots=\beta_{k}=0, \quad$ then $\frac{S_{T}^{2}}{\sigma^{2}} \sim \chi^{2}(n-1)$ and $\frac{S_{R}^{2}}{\sigma^{2}} \sim \chi^{2}(k)$ is true.

The above property shows that the identified $S_{T}^{2}$ should be small if $\mathrm{H}_{0}$ is true and that large $\frac{S_{R}^{2}}{S_{E}^{2}}$ is a low-probability event. The rejection region form is $\left\{\frac{S_{R}^{2}}{S_{E}^{2}}>c\right\}$. Therefore, if $\mathrm{H}_{0}$ is correct, the critical value of the given significant level $\alpha$ is

$$
c=\frac{k}{n-k-1} F_{1-\alpha}(k, n-k-1)
$$

When $y$ and $X_{1}, X_{2}, \ldots, X_{k}$ have a significant linear relationship, the significance of each variable $X_{i}(i=1,2, \ldots, k)$ should be verified. If $X_{i}$ exerts no significant effect on $Y$, then $\beta_{i}$ should be zero, that is, $\mathrm{H}_{0 i}: \beta_{i}=0(i=1,2, \ldots, k)$ should be tested. Its rejection region form is $\left\{\left|\beta_{i}\right|>c_{i}\right\}$, where

$c_{i}=S_{E} \sqrt{\frac{c_{i i} F_{1-\alpha}(1, n-k-1)}{n-k-1}}, c_{i i}=\operatorname{diag}\left(\left(X^{\mathrm{T}} X\right)^{-1}\right)$.
After substituting the stress time history data, relevant load time history data, and optimized coefficient of D46 into the above equation, the following can be obtained.

$$
\begin{aligned}
& \frac{S_{R}^{2}}{S_{E}^{2}}=19.56, \\
& c=\frac{8}{10485927} F_{1-\alpha}(8,10485927)=1.929 E-06 .
\end{aligned}
$$

If $\frac{S_{R}^{2}}{S_{E}^{2}}>c$ then it falls in the $\mathrm{H}_{0}$ rejection region; this condition indicates that the linear relationship coefficient of all $X$ and $Y$ cannot be zero. Thus, a linear relationship is established.

Each linear coefficient influences the significance level, which is presented in Table 5 .

$\beta_{i}>c_{i},(i=1, \ldots, 8)$, indicating that $\mathrm{H}_{0 i}(i=1, \ldots, 8)$ falls in the rejection region, and all variables $\left(x_{1}, x_{2}, x_{3}, x_{4}, x_{5}, x_{6}\right.$, $x_{7}, x_{8}$ ) have a significant linear impact on $y$.

\section{Prediction of Stress at the Speed of $200 \mathrm{~km} / \mathrm{h}$}

After obtaining the optimized transitive relation in the test run at an operating speed of $160 \mathrm{~km} / \mathrm{h}$, the load data obtained in a formal test at a speed of $200 \mathrm{~km} / \mathrm{h}$ can be utilized to predict stress data. The coefficient calibrated in

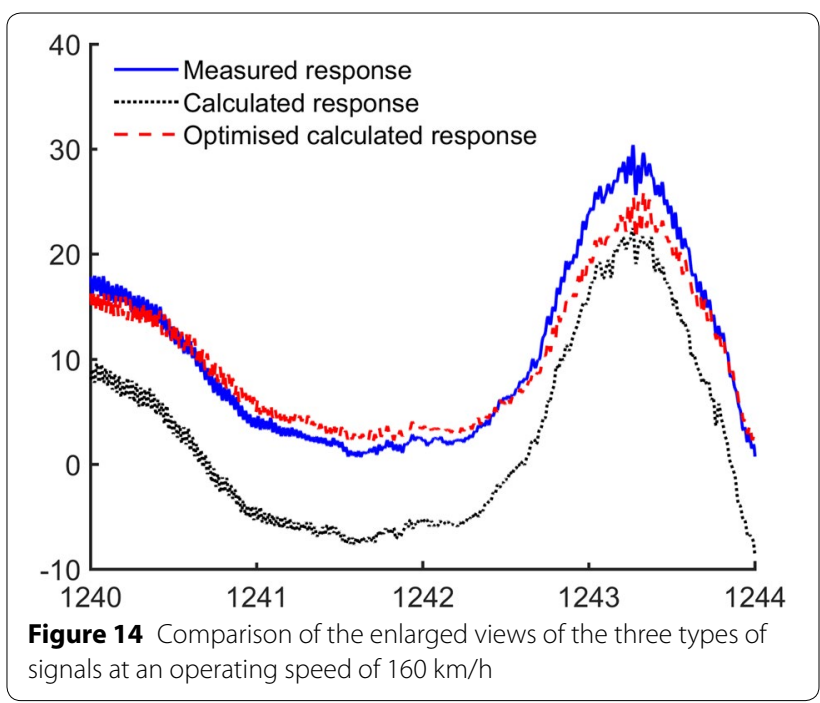

Table 4 Calculated stress values of modified transitive relation and actual measured signal error

\begin{tabular}{lllllllll}
\hline Error & \multicolumn{3}{l}{ Measuring points } & & & \\
\cline { 2 - 8 } & D13 & D15 & D26 & D34 & D46 & D48 & D55 & D57 \\
\hline Mse2 & 0.06 & 0.14 & 0.20 & 0.11 & 0.06 & 0.08 & 0.13 & 0.11 \\
\hline
\end{tabular}

Mse2 represents the percentage mean square error between the stress value calculated with Eq. (10) and the measured stress value 
Table 5 Each linear coefficient influences the significance level $1 \times 10^{-2}$

\begin{tabular}{rrrrrrrrr}
\hline $\boldsymbol{i}$ & $\mathbf{1}$ & $\mathbf{2}$ & $\mathbf{3}$ & $\mathbf{4}$ & $\mathbf{5}$ & $\mathbf{6}$ & $\mathbf{7}$ & $\mathbf{8}$ \\
\hline$c_{i}$ & 0.02 & 0.22 & 0.12 & 0.2 & 0.01 & 0.02 & 0.004 & 0.002 \\
$\beta_{i}$ & 12.42 & 18.51 & 43.29 & -462.01 & -3.03 & -44.23 & -0.14 & -0.37 \\
\hline
\end{tabular}

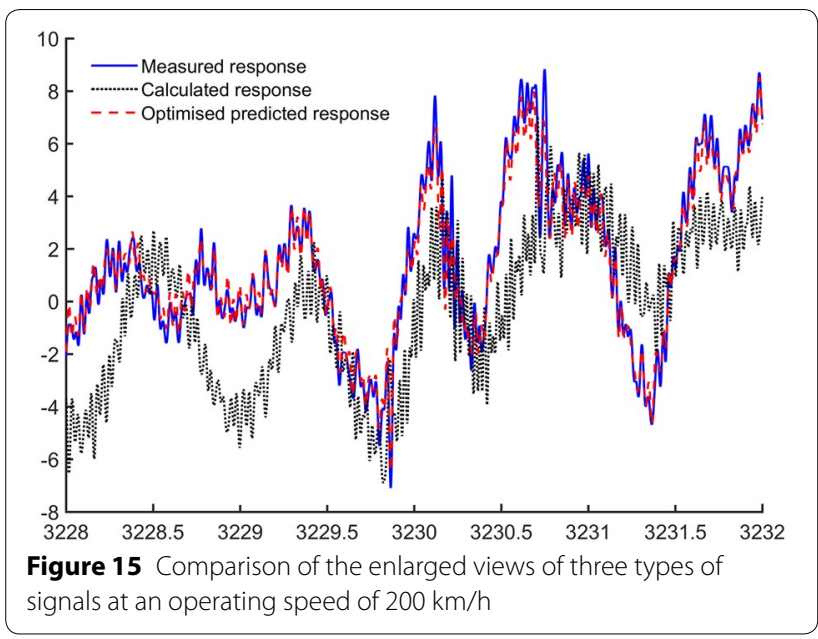

the laboratory and the actual measured signal can be used for comparison. The local area model is zoomed in in Figure 15 . The stress data predicted by the modified transitive relation and measured signal error are shown in Table 6.

\section{Comparison of Multiple Working Conditions and Analysis}

After modifying the transitive relation between load and stress, the load data of the intercity EMU at different working conditions, such as entering or departing the depot, upgoing line, downgoing line, and heavy vehicle load, are substituted into the aforementioned transitive relation and the transitive relation calibrated in the laboratory. Two calculated stress errors at the measuring points are shown in Tables 7 and 8.

As shown in Tables 7 and 8, all the stress value errors calculated with the optimized transitive relation are smaller than the stress value errors obtained with the laboratory-calibrated transitive relation. The optimized transitive relation can truly reflect the load-stress transitive relation of the bogie frame under the constraints of line operation. The data in Tables 7 and 8 also indicate that the modified load-stress transitive relation does not significantly vary in different working conditions.

The laboratory-calibrated load-stress transitive relation differs from the real load-stress transitive relation. This difference is caused by the difference between the constraints on the road and those on the laboratory bench, even if the laboratory bench installs the bogie, including spring, axle box, rod, anti-yaw bracket, and other related accessories, according to actual operating conditions. The most significant influencing factors are rigidity matching and gap issues. Different constraint stiffness values and contact gaps in the same direction may offset or increase the stress caused by different loads. Another cause for such a difference is the effect of the changing wheel-rail

Table 6 Stress predicted by modified transitive relation and measured signal error at an operating speed of $200 \mathrm{~km} / \mathrm{h}$

\begin{tabular}{|c|c|c|c|c|c|c|c|c|}
\hline \multirow[t]{2}{*}{ Error } & \multicolumn{8}{|c|}{ Measuring points } \\
\hline & D13 & D15 & D26 & D34 & D46 & D48 & D55 & D57 \\
\hline Mse2 & 0.06 & 0.12 & 0.14 & 0.05 & 0.06 & 0.07 & 0.10 & 0.10 \\
\hline Mse1 & 0.34 & 1.92 & 1.59 & 1.65 & 2.32 & 1.19 & 0.78 & 1.38 \\
\hline
\end{tabular}

Table 7 Measured values and the calculated value errors of the laboratory-calibrated coefficients under multiple working conditions (Mse1)

\begin{tabular}{|c|c|c|c|c|c|c|c|c|}
\hline \multirow[t]{2}{*}{ Error } & \multicolumn{8}{|c|}{ Measuring point } \\
\hline & D13 & D15 & D26 & D34 & D46 & D48 & D55 & D57 \\
\hline Entering or departing the depot calculation error & 0.09 & 1.17 & 0.80 & 1.02 & 0.86 & 1.52 & 0.21 & 0.99 \\
\hline Upgoing line working condition calculation error & 0.29 & 1.96 & 1.38 & 1.59 & 1.74 & 1.59 & 0.73 & 1.14 \\
\hline Downgoing line working condition calculation error & 0.31 & 1.89 & 1.43 & 1.66 & 1.84 & 1.57 & 0.71 & 1.12 \\
\hline Heavy vehicle working condition calculation error & 0.30 & 2.13 & 1.81 & 2.00 & 2.36 & 1.81 & 0.95 & 1.35 \\
\hline
\end{tabular}


Table 8 Measured values and calculated value errors of the optimized transitive relation coefficients under different road conditions (Mse2)

\begin{tabular}{|c|c|c|c|c|c|c|c|c|}
\hline \multirow[t]{2}{*}{ Error } & \multicolumn{8}{|c|}{ Measuring point } \\
\hline & D13 & D15 & D26 & D34 & D46 & D48 & D55 & D57 \\
\hline Entering or departing the depot working condition calculation error & 0.03 & 0.06 & 0.08 & 0.02 & 0.05 & 0.03 & 0.04 & 0.02 \\
\hline Upgoing line working condition calculation error & 0.10 & 0.21 & 0.14 & 0.08 & 0.31 & 0.07 & 0.10 & 0.10 \\
\hline Downgoing line working condition calculation error & 0.06 & 0.12 & 0.14 & 0.05 & 0.06 & 0.07 & 0.10 & 0.106 \\
\hline Heavy vehicle working condition calculation error & 0.03 & 0.10 & 0.29 & 0.08 & 0.04 & 0.23 & 0.11 & 0.12 \\
\hline
\end{tabular}

contact point on the arm of force. Therefore, from a statistical perspective, the optimized load-stress transitive relation is more reasonable than that calibrated by laboratory bench. The stress calculated by the optimized transfer coefficient still exhibits a certain degree of deviation from the measured signal. Such deviation is mainly caused by partial loads. For example, primary vertical damper loads and secondary horizontal damper loads are not measured in this test. Another reason is that elastic vibration phenomenon exists in the local structure (e.g., motor bracket). Overall, load and stress exhibit a linear relationship under the operating conditions.

The frequency-domain analysis indicates that when elastic vibration does not occur, the transitive relation can be described as a linear transitive relation, namely, $x=\Sigma K_{i} F_{i}$, where $F_{i}$ denotes the load, $K_{i}$ denotes the transitive relation coefficient, and $x$ denotes the response strain. The linear relationship reflects the transitive relation between the load and stress of the vehicle under such assembly process conditions. When the line conditions deteriorate and the load changes, the strain response will change accordingly. When the vehicle weight increases and the load increases, the strain response will also increase. The linear relationship significance test and the comparison of data under different working conditions also prove that a linear transitive relation exists and remains unchanged.

\section{Conclusion}

1. Spectrum analysis of the load and the stress signal indicated that the main frequency components of the intercity bogie frame are $20 \mathrm{~Hz}$ to $60 \mathrm{~Hz}$.

2. An optimized nonlinear model and a linear model were compared in terms of load and stress data from a large sample. With the use of a BP neural network, the fitting error of the linear transitive relation was smaller than the nonlinear fitting error.

3. A simplified neural network model can represent the transitive relation between load and stress. The stress value error calculated with the transitive relation under different operating conditions was smaller than the stress value error calculated with the cali- brated transitive relation on the laboratory bench. This finding indicates that the modified transitive relation did not vary significantly under various working conditions.

4. The verification and validation of the linear model provide strong support for the linear superposition of the load signal. The modified transitive relation can be utilized to calculate the damage consistency and infer the time history signal of the load according to the time history signal of stress.

Authors' Contributions

$\mathrm{HZ}$ was in charge of the whole trial; HZ wrote the manuscript; S-GS, QL, Z-SR assisted with sampling and laboratory analyses. All authors have read and approved the final manuscript.

\section{Authors' Information}

Hua Zou, born in 1975, is currently a lecturer in Beijing Jiaotong University, China. He received his PhD degree from Beijing Jiaotong University, China, in 2016. His research interests include structural strength and experimental research of structural dynamic characteristics. Tel: +86-10-51683195; E-mail: hzou@bjtu.edu.cn.

Shou-Guang Sun, born in 1962, is currently a professor in Beijing Jiaotong University, China. He received his PhD degree from Tsinghua University, China, in 1992. His research interests include structural fatigue, fracture and reliability. E-mail: shgsun@bjtu.edu.cn.

Qiang Li, born in 1963, is currently a professor in Beijing Jiaotong University, China. He received his PhD degree from Beijing Jiaotong University, China, in 1995. His research interests include structural fatigue, fracture and reliability. E-mail: qli3@bjtu.edu.cn.

Zun-Song Ren, born in 1969, is currently a professor in Beijing Jiaotong University, China. He received his PhD degree from Southwest Jiaotong University, China, in 2000. His research interests include vehicle system dynamics and structural reliability. E-mail: zsren@bjtu.edu.cn.

\section{Competing Interests}

The authors declare no competing financial interests.

Ethics Approval and Consent to Participate

Not applicable.

\section{Funding}

Supported by National Natural Science Foundation of China (Grant No. U1134201), National Key Research and Development Program of China (Grant No. 2016YFB1200404-08), and National Key Research and Development Program of China (Grant No. 2016YFB1200505-11).

\section{Publisher's Note}

Springer Nature remains neutral with regard to jurisdictional claims in published maps and institutional affiliations. 
Received: 8 February 2016 Accepted: 16 March 2018

Published online: 11 April 2018

\section{References}

[1] S G Zhang. Study on testing and establishment method for the load spectrum of bogie frame for high-speed trains. Science China Series E: Technological Sciences, 2008, 51: 2142-2151.

[2] Y J Wei. Research on the mechanics of high speed rails. Acta Mechanica Sinica, 2016, 32(2): 1-2

[3] N Zhu, S G Sun, Q Li, et al. Theoretical research and experimental validation of load spectra on bogie frame structures of high-speed trains. Acta Mechanica Sinica, 2014, 30(6): 901-909.

[4] W Wang, Y Wang, S Sun, et al. Long-term load spectrum test of highspeed train bogie. Journal of Southwest Jiaotong University, 2015, 50(1): 84-89. (in Chinese)

[5] N Zhu, S G Sun, Q Li, et al. Theoretical research and experimental validation of elastic dynamic load spectra on bogie frame of high-speed train. Chinese Journal of Mechanical Engineering, 2016, 29(3): 498-506.

[6] Z S Ren, S G Sun, Q Li, et al. Experimental studies of load characteristics of bogie frames for $350 \mathrm{~km} / \mathrm{h}$ EMUs. Proceedings of the Institution of Mechanical Engineers Part F Journal of Rail \& Rapid Transit, 2012, 226(2): 216-227.

[7] C J Mi, Z Q Gu, W G Wu, et al. Fatigue life analysis of rear axle housing of mining dump truck under random load. Journal of Mechanical Engineering, 2012, 48(12): 103-109. (in Chinese)

[8] W H Ma, S H Luo, R R Song. Load spectrum research of tumbler journal box node of high-speed motor car. Railway Locomotive \& Car, 2009, 29(4): 12-14. (in Chinese)

[9] P Wolfsteiner, W Breuer. Fatigue assessment of vibrating rail vehicle bogie components under non-Gaussian random excitations using power spectral densities. Journal of Sound \& Vibration, 2013, 332(332): 5867-5882.

[10] HYYe. The dynamic stress test of random load loading on high speed bogie frame. Chengdu: Southwest Jiaotong University, 2011. (in Chinese)

[11] D F Zhang. The load spectrum study on high speed bogie frame. Chengdu: Southwest Jiaotong University, 2013. (in Chinese)

[12] Z S Ren, S G Sun, Q Li. Axle spring load test and dynamic characteristics analysis of high speed EMU. Journal of Mechanical Engineering, 2010, 46(10): 109-115. (in Chinese)

[13] D G Liu, L G Shao. Consideration on the effect of the elasticity of car-body and frame on the dynamic load, dynamic stress and fatigue damage. Rolling Stock, 2005, 45: 1-4. (in Chinese)
[14] Z S Ren, S G Sun, Q Li, et al. Simulation of railway bogie dynamic stress and elastic vibration. Journal of Mechanical Engineering, 2004, 40(8): 187-192. (in Chinese)

[15] W J Wang, S G Sun, Q Li. Dynamic stress simulation of flexible bogie frame. Journal of the China Railway Society, 2006, 28(1): 44-49. (in Chinese)

[16] CW Liang. Dynamic analysis of the geometrical nonlinear model derived from bogie. Harbin: Harbin Institute of Technology, 2012.

[17] A H Wickens. Static and dynamic instabilities of bogie railway vehicles with linkage steered wheelsets. Vehicle System Dynamics, 1996, 26(1): $1-16$

[18] R V Dukkipati, S Narayanaawamy, M O M Osman. Comparative performance of unconventional railway trucks. International Journal of Vehicle Design, 1998, 19(3): 326-339.

[19] Z S Ren. Multi-point contact of the high-speed vehicle-turnout system dynamics. Chinese Journal of Mechanical Engineering, 2013, 26(3): $518-525$

[20] K G Liu, C L Yan, S M Zhang. Bench calibration of load spectrum measurement for fighter airplane main undercarriage. Journal of Jilin University (Engineering and Technology Edition), 2006, 36: 1025-1028. (in Chinese)

[21] W M Zhai, P Liu, J Lin, et al. Experimental investigation on vibration behaviour of a $\mathrm{CRH}$ train at speed of $350 \mathrm{~km} / \mathrm{h}$. International Journal of Rail Transportation, 2015, 3(1): 1-16.

[22] X C Jin, S G Sun, G X Chen. Study on vertical dynamic response of railway track induced by express trains. China Safety Science Journal, 2005, 15: 38-42. (in Chinese)

[23] L Chi, J Liu, C Jiang. Radial basis shape function method for identification of dynamic load in time domain. China Mechanical Engineering, 2013, 24(3): 285-289. (in Chinese)

[24] X Han, J Liu, W J Li, et al. A computational inverse technique for reconstruction of multisource loads in time domain. Chinese Journal of Theoretical and Applied Mechanics, 2009, 41 (4): 595-602. (in Chinese)

[25] M Chen. The MATLAB neural network theory and examples. Beijing: Tsinghua University Press, 2013. (in Chinese)

[26] J F Qiao, H G Han. Analysis and design of feedforward neural networks. Beijing: Science Publishing House, 2013. (in Chinese)

[27] H Yang, Q S Liu. Mathematical statistics. Beijing: Higher Education Press, 2004. (in Chinese)

\section{Submit your manuscript to a SpringerOpen ${ }^{\circ}$ journal and benefit from:}

- Convenient online submission

- Rigorous peer review

- Open access: articles freely available online

- High visibility within the field

Retaining the copyright to your article

Submit your next manuscript at springeropen.com 